\title{
Renta Básica y Renta Máxima: una concepción republicano-democrática*
}

\author{
Basic Income and Maximum Income: a republican \\ democratic conception
}

\author{
MARÍA JULIA BERTOMEU ** \\ DANIEL RAVENTÓS ***
}

\begin{abstract}
Resumen:. El propósito del presente trabajo es i) de fundamentar y mostrar la viabilidad de una Renta Básica, entendida como resguardo de un mínimum de existencia social para todos, que permitiría garantizar una universalización de la libertad republicana; y también ii) abordaremos el tema de la justificación de una Renta Máxima (RM), entendiendo que la acumulación de riqueza y, por ello mismo, de poder económico y político de los muy ricos, atenta contra el deber fiduciario de las repúblicas democráticas de garantizar una "vida normal" para todos sus habitantes.

Palabras clave: Renta Básica, Renta Máxima, libertad republicana, propiedad
\end{abstract}

\begin{abstract}
Our aims are 1) to establish and demonstrate the viability of a basic income, which is understood as safeguarding a minimum of social existence for everyone, and that would guarantee the universalisation of republican freedom; and 2) to tackle the question of justifying a Maximum Income, in the understanding that the accumulation of wealth, and hence of the political and economic power of the very rich, is an assault against the fiduciary duty of democratic republics to guarantee a "normal" life for all citizens.
\end{abstract}

Key words: Basic income, Maximum income, Republican freedom, Property.

Recibido: 18/05/2020. Aceptado: 19/06/2020. Cómo citar este articulo: Bertomeu, M.J.; Raventós, D. (2020). Renta Básica y Renta Máxima: una concepción republicano-democrática. Daimon. Revista Internacional de Filosofía, (81), 197-213. https://doi.org/10.6018/daimon.428401

* Este trabajo se ha desarrollado en el marco del proyecto PGC2018-094324-B-I00 (MCIU/AEI/FEDER, UE).

** Investigadora Principal del Consejo Nacional de Investigaciones Científicas de Argentina (INEO-CIF). Correo electrónico: mjbertomeu@gmail.com. Ultimas publicaciones: "La concepción republicana y fiduciaria de la propiedad y de la soberanía política, y sus consecuencias para la universalización de la libertad no dominada". (2020) en prensa. Kant ¿liberal o republicano? Con-Textos-Kantianos (2019).

*** Economista, profesor titular del departamento de Sociología de la Facultad de Economía y Empresa de la Universidad de Barcelona. Correo electrónico: danielraventos@ub.edu. Es coautor, junto con Jordi Arcarons y Lluís Torrens, de Renta Básica Incondicional. Una propuesta de financiación racional y justa (Serbal, 2017) y, en colaboración con Julie Wark, Against Charity (Counterpunch, 2018) traducido al castellano (Icaria) y al catalán (Arcadia). 


\section{Introducción}

En una entrevista realizada por Maciej Szlinder a Antoni Domènech publicada en el año 2018, Domènech -asiduo y convencido fiador de una concepción republicana y por eso mismo también socialista de la Renta Básica-, expresó una idea interesante sobre ambas rentas - la básica y la máxima- en relación con la vida política cotidiana en una república democrática a la que dio en llamar "normal":

“(...) pocas cosas -si es que hay alguna que pudiera hacerlo- contribuirían tanto hoy a mitigar la capacidad de los imperios privados para desafiar con éxito a los poderes públicos democráticos como una renta básica de ciudadanía que, al tiempo que exigiera un minimum de existencia social para todos, se batiera también por limitar a un maximum compatible con la vida política republicano-democrática normal el volumen de ingresos que le es dado recibir a un ciudadano. Una milenaria sabiduría política mediterránea, retrotraíble a Solón o a los hermanos Graco, enseña lo que famosamente repitió muchos siglos después el mejor Maquiavelo: que una vida política libre, republicana, es incompatible con la existencia de magnates" (Szlinder, $2018,35) \cdot{ }^{1}$

A modo de homenaje a quien fue nuestro compañero, amigo y maestro, nos interesa abundar sobre esta jugosísima cita, con las herramientas histórico-conceptuales propias de un programa de investigación sobre "las raíces históricas y conceptuales del republicanismo democrático clásico" que Domènech generosamente nos legó a muchos de nosotros, también valedero para el momento actual del modo de producir capitalista en el que nos encontramos al inicio de la tercera década del siglo XXI.

La marcha de nuestra exposición recorrerá el siguiente camino. En primer lugar i) proponemos una muy breve exposición sobre los fundamentos y la viabilidad de una Renta Básica (RB) entendida como resguardo de ese mínimum de existencia social para todos, que permitiría garantizar una universalización de la libertad republicana; en segundo lugar ii) abordaremos el tema de la justificación de una Renta Máxima (RM), entendiendo que la acumulación de riqueza y, por ello mismo, de poder económico y político de los muy ricos, atenta contra el deber fiduciario de las repúblicas democráticas de garantizar una "vida normal" para todos sus habitantes. Por último iii) unas breves conclusiones.

En un trabajo publicado en el año 2005, firmado en coautoría por Antoni Domènech y María Julia Bertomeu, se propuso una definición de la libertad republicana histórica dentro de la vida social. Especialmente importantes para el presente trabajo son dos rasgos que

1 Aunque más adelante se ofrece una definición más extensa, adelantamos ahora que por Renta Básica entendemos una asignación monetaria pública incondicional y universal; y por Renta Máxima una cantidad de riqueza determinada a partir de la cual no estaría legalmente autorizado acumular más. O lo que es lo mismo: una tasa marginal impositiva del 100\%. Al hablar de una Renta Básica con fundamentación republicanodemocrática y, por lo mismo, también socialista, hacemos nuestra la idea domenechiana de que buena parte del socialismo político -y también el anarquismo- arrancó en Europa de la mano de la democracia republicana heredera del ideario jacobino, y que incluso Marx y Engels presentaron en su Manifiesto de 1848 al comunismo como herencia de esa democracia social revolucionaria. Sobre esta tesis: Domènech (2019). 
-junto con otros- integran dicha definición y que ahora hacemos nuestros. El primero, que es el núcleo de la fundamentación republicano-democrática de la Renta Básica que proponemos: i) que es republicanamente libre quien no depende de otro particular para vivir, es decir, el que goza de una existencia social autónoma garantizada, porque tiene algún tipo de propiedad que le permite subsistir bien, sin tener que pedir cotidianamente permiso a otros. Y el segundo, con el que ensayaremos argumentar a favor de una Renta Máxima: ii) que la república está obligada a interferir en el ámbito de existencia social privada de $\mathrm{X}$, si ese ámbito privado capacita a X para disputar con posibilidades de éxito a la república el derecho de ésta a garantizar qué sea el bien común.

\section{La Renta Básica como garantía universal de una existencia social autónoma}

Proponemos ordenar este apartado en torno a la siguiente cuestión: ¿por qué insistir en una RB para lograr la universalización de la libertad republicana? ¿qué sentido tiene perseverar con la libertad cuando lo que hoy preocupa fundamentalmente es la desigualdad, la injusticia y la solidaridad?

Sabemos que actualmente se ensayan muy distintas maneras de fundamentación de la $\mathrm{RB}$, aunque no todas ellas resultan compatibles con el diseño de una república democrática comprometida con la existencia material de toda la ciudadanía. Huelga decir que las propuestas conservadoras que defienden a viva voz una RB para terminar con los restos de los Estados de bienestar de postguerra son incompatibles con una democracia económica, entre otras cosas porque se sirven de esta propuesta para liquidar de un plumazo los derechos duramente conquistados por la mayoría de los explotados de la tierra, como lo son el derecho a la salud, educación, vivienda y salario dignos y la legislación laboral, entre otros.²

Los autores de este artículo estamos convencidos de que una RB capaz de garantizar el derecho incondicional a una existencia social autónoma es una herramienta -normativa y práctica- muy potente para el diseño de una república democrática, incluso en las condiciones sociales contemporáneas de crisis capitalista agravada por la pandemia del Coronavirus (Covid-19), que pueden conllevar una crisis económica y social de una magnitud desconocida en la historia de los siglos XX y XXI. También pensamos que algunos de los conceptos propios de la filosofía política republicana permiten pensar una RB entendida como una propuesta -no la única- de diseño institucional para promover la universalización de una libertad robusta republicana que, para abreviar, llamaremos no dominada. Y ante la pregunta de por qué insistir en hablar sólo de la libertad si lo que ahora toca es hablar de desigualdad, en vista de las estadísticas de su crecimiento exponencial, nuestra respuesta es -lo decimos con una expresión acertada de Clare Roberts cuando le preguntaron si Marx estaba interesado en la libertad o meramente en la igualdad-que estamos interesados en el goce igual de una libertad no dominada, y que una RB a un nivel adecuado podría contribuir a alcanzarla, junto con otras medidas republicanas y muy especialmente con un diseño republicano democrático del instituto jurídico de la propiedad, pública y privada (Roberts, 2017).

2 El libro de uno de los más conocidos defensores de la Renta Básica desde una posición neoliberal, Charles Murray (2006), es suficientemente elocuente: In Our Hands: A Plan To Replace The Welfare State. 
En este apartado abundaremos en torno a algunos argumentos para la defensa de una RB capaz de garantizar la universalización de la libertad no dominada, por la vía de garantizar derechos constitutivos ex ante y de un diseño institucional acorde con tales garantías. Comencemos con unas muy breves pinceladas en torno a la definición de una RB, para luego ocuparnos de su fundamentación republicana $\mathrm{y}$, finalmente, de una propuesta de posible financiación.

Hay varias definiciones de RB, aunque a nuestro entender la siguiente es la mejor por lo clara y provocadora, a pesar de ser larga:

"La renta básica es un ingreso pagado por el estado a cada miembro de pleno derecho de la sociedad o residente ${ }^{3}$, incluso si no quiere trabajar de forma remunerada, sin tomar en consideración si es rico o pobre, o dicho de otra forma, independientemente de cuáles puedan ser las otras posibles fuentes de renta, y sin importar con quién conviva." 4

Dicho someramente, es un ingreso que paga el estado de manera individual a todos y sin condiciones, sin caer en los prejuicios y motes que se imponen a quienes no están dispuestos a aceptar un cierto tipo de trabajo remunerado indigno, ni favorece algún tipo de convivencia. Vayamos ahora a su fundamentación, sin olvidar que es necesario -ahora y siempreluchar por una garantía similar a una Renta Básica para "la familia humana” en su conjunto.

Asistimos hoy a un oportuno redescubrimiento del concepto de libertad republicana entendida como no dominación, probablemente a causa de la impotencia de las herramientas usadas por el grueso del liberalismo de izquierda -anglosajón, aunque preferentemente norteamericano- cuando se trata de abordar las cuestiones de la injusticia, desigualdad e inequidad de las democracias contemporáneas; aunque también a causa de la transformación neoliberal ${ }^{5}$ de lo que en algún momento dio en llamarse "liberalismo social" y de la

3 Somos conscientes de que al hablar de "miembro de pleno derecho de la sociedad o residente" no se incluye como beneficiario de una RB a la inmensa mayoría de inmigrantes que, desamparados y abandonados, huyen de sus países a causa de las crisis económicas, políticas, ecológicas y de los conflictos bélicos que la mayoría de las veces provocan los países que ahora los condenan a la muerte. Muy lejos de desconocer el problema, creemos que es preciso abordar este tema de manera realista en el marco de la tan olvidada, pero potente Declaración de derechos humanos de post-guerra, que hoy cobra renovada vigencia ante la pandemia del coronavirus que azota a la humanidad en medio de una crisis capitalista profunda. El primer considerando del "Preámbulo" de 1948 dice -un decir que estuvo a punto de hacer fracasar la declaración por las marcadas diferencias político filosóficas entre sus miembros- que: "la libertad, la justicia y la paz en el mundo tienen por base el reconocimiento de la dignidad intrínseca de los derechos iguales e inalienables de todos los miembros de la familia humana". Hoy más que nunca deberíamos recordar la metáfora conceptual de la familia humana entendida como la unión fraternal (y por eso mismo horizontal) entre los seres humanos iguales en derechos y en dignidad (artículo 1); metáfora que cobra fuerza, también, al hablar de seres humanos que en todas partes tienen derecho al reconocimiento de su personalidad jurídica (artículo 6). Afirmar que en todas partes los seres humanos tienen derecho al reconocimiento de su personalidad jurídica -a realizar actos y negocios jurídicos y contraer derechos y obligaciones adquiridas por sí mismos y sin depender de otros-, implica conferir a la humanidad en su conjunto (al menos como proyecto político confesado) el carácter de sui iuris, de sujetos de derechos o, lo que es lo mismo, de sujetos libres republicanamente. Una RB de carácter universal sería una herramienta para lograrlo.

4 Para una explicación exhaustiva de esta definición, de sus antecedentes históricos, críticas y contra-críticas, Raventós (2007).

5 La palabra "neoliberal" se ha hecho ubicua y no siempre se utiliza con el mismo significado. Cuando nosotros la usamos en este texto, entendemos aquella fase del capitalismo iniciada en la década de los 70 del siglo pasado 
conversión de grandes partes de la actual socialdemocracia europea al social-liberalismo, partidario de las reformas del Estado de Bienestar de postguerra.

En los últimos años -y como alternativa ante un liberalismo político que va perdiendo su cetro en la academia-, ha ganado terreno la opción neo-republicana y neo-romana de Quentin Skinner y Philip Pettit; aunque también una versión republicana radical, que brega por recuperar un lenguaje republicano heredado por el primer socialismo y que, entre otras cosas, hizo posible elaborar una crítica a la supuesta condición de libre del trabajo asalariado, en condiciones de apropiación indebida de los medios de producción. A diferencia de Pettit y de los pettitianos, las versiones republicanas radicales hacen hincapié en el carácter estructural o sistémico de la dominación, y no sólo en una supuesta dominación personal entre agentes intencionales con distinto poder. ${ }^{6}$ Varios historiadores y filósofos políticos republicanos contemporáneos intentan diferenciarse del republicanismo romano "aristocrático" por distintas vías, entre ellas, la de recuperar los grandes movimientos de emancipación del socialismo norteamericano de finales del XIX, como es el caso de Alex Gourevitch quien, dicho sea de paso, es un crítico acérrimo de la Renta Básica; o como lo hace Clare Roberts cuando reconstruye a Marx con la mirada de un explorador decidido a descubrir su filiación con el proyecto republicano radical de garantizar universalmente la libertad igual.

Siguiendo la línea de trabajos anteriores publicados por los autores del presente escrito -por separado y de consuno- propondremos ahora una lectura republicana de la Renta Básica que abreva de manera directa en el núcleo republicano del iusnaturalismo revolucionario clásico, renacido en la célebre Declaración de Derechos de 1948, luego de más de un siglo de eclipse. No es en vano recordar, por ejemplo, que el primer considerando del preámbulo de la Declaración de 1948 afirma enfáticamente, que la libertad ha de ser universalizada a "todos" y que es inalienable, y que la "igualdad" bien entendida dimana de la libertad bien entendida. Sin duda alguna se trata de un manifiesto republicano según el cual la igualdad no es otra cosa que la universalización de la libertad no dominada. ${ }^{7}$ Si bien la Declaración no se detiene a profundizar expresamente los alcances jurídicos de la inalienabilidad de los derechos iguales de todos, alegar que ciertos derechos son inalienables e iguales es toda una manifestación de principios y de compromisos con una tradición histórica de larga data.

Si la libertad es inalienable, entonces el conjunto de derechos que la configuran son constitutivos, no pueden venderse ni tampoco regalarse. Todo esto muestra que las raíces del derecho público moderno son republicanas, pues poco o nada tendría para decir un liberalismo partidario de una concepción de la libertad como mera minimización de la interferencia (actual y real) ante aquellas decisiones privadas de los individuos que no afectan a terceros. La libertad sólo sería concurrente con un mundo de seres humanos liberados del temor y la miseria.

que se caracteriza por "políticas de austeridad, una profunda desigualdad, el dominio del capital financiero y la concentración de poder de mercado en pocas corporaciones" (Nadal, 2019).

6 Hay una larga lista de autores que han comenzado a recuperar lo que podemos llamar una versión radical de la tradición republicana, además de los ya citados y, entre ellos, el de Antoni Domènech y los miembros de su escuela. También otros autores como Gourevitch (2015), Gauthier (1992), Hammel (2019), abonan una recuperación del republicanismo en su versión plebeya.

7 Para una lectura republicana de la Declaración de Derechos de 1948, véase Bertomeu (2016). 
Pero los temores y las miserias están históricamente indexados; en 1948 se trataba de terminar con una política mundial fundada en el nacionalismo imperialista, el colonialismo y el racismo, y perpetradora de masacres que dieron origen al término genocidio. En este momento los temores y las miserias son otros: una buena parte de los miembros de la familia humana han sido despojados de su personalidad jurídica, sin derecho a una nacionalidad, a circular libremente, a participar en el gobierno de su país. Pero además, la mayor parte de la población no rica aún no ha conseguido los niveles sociales y económicos de antes de la crisis de 2008, una nueva crisis golpeará a esta población. Es tiempo de medidas incondicionales y universales, para eliminar los errores de exclusión y las demoras, y abonar una cohesión social posible en momentos de crisis. Nuevamente la $\mathrm{RB}$ puede ser un instrumento.

\section{Renta Básica. Una propuesta de financiación}

A finales de 2017 se publicó un estudio fruto de varios años de elaboración, ${ }^{8}$ sobre la manera en que se podría financiar una RB en el Reino de España. Este estudio pretende mostrar cómo - a partir de una base de datos inmensa sobre el Impuesto de la Renta de las Personas Físicas (IRPF)- se puede financiar una RBde una cantidad igual al umbral de la pobreza, para todas las personas residentes en el Reino de España. Lo que pretendemos aquí es simplemente aportar los aspectos fundamentales muy resumidos de este estudio.

La base de datos de la que se partía fue una muestra cedida por el Instituto de Estudios Fiscales, de cerca de dos millones de liquidaciones del impuesto de la renta de las personas físicas (IRPF) de todo el Reino de España, excepto la Comunidad Autónoma Vasca (CAV) y Navarra. ${ }^{9}$ Los datos eran del año 2010, puesto que se pretendía hacer el estudio en un año inmerso en plena crisis. Disponemos hoy de nuevos datos, pero no harán variar excesivamente los resultados dada la poca variabilidad del IRPF ante los cambios coyunturales, ${ }^{10} \mathrm{a}$ diferencia, por ejemplo, del impuesto de sociedades.

Esta información permitía una imputación prácticamente exhaustiva de todo el flujo de rendimientos netos de la persona declarante en el IRPF: trabajo, capital mobiliario, inmobiliario, actividades económicas, ganancias y pérdidas patrimoniales, así como identificar las características socio-familiares de la persona declarante: edad, estado civil, descendientes, ascendientes, declaración individual o conjunta, etc.

De los 43,7 millones de personas que deberían ser perceptoras de la RB, se detectan en el IRPF que se maneja 34,3 millones. No se identifican dentro del IRPF exactamente 9,4 millones de personas (8,2 adultas y 1,2 menores).

8 Arcarons, Raventós y Torrens (2017). Este apartado sintetiza fundamentalmente la investigación citada que tuvo un avance en 2014: Arcarons, Domènech, Raventós y Torrens (2014).

9 Las razones de esta exclusión son simplemente técnicas, puesto que estas comunidades al no estar dentro del llamado sistema común, podían distorsionar algunos datos. Un estudio con la misma técnica para la provincia de Guipúzcoa, no ya con una muestra sino con la totalidad de IRPFs de aquella provincia se puede descargar en http://www.redrentabasica.org/rb/rrbantigua_1107/ (último acceso 17 de mayo de 2020).

10 Siendo esto cierto en general, la situación extraordinaria producida por la pandemia y la semiparalización de gran parte de la economía mundial, puede modificar esta afirmación. 
El IRPF de partida muestra una importante desigualdad de renta. Así, el índice de Gini de la Renta Bruta (saldo neto de rendimientos sin posibles compensaciones a la que debe añadirse las ganancias patrimoniales), antes por tanto de la declaración de IRPF, es de 0,4114. $\mathrm{Y}$ el posterior (renta bruta menos cuota IRPF) es de 0,3664. Es decir, el IRPF solamente corrige el índice de Gini en menos de un $5 \%(0,4114$ - 0,3664 = 0,045). Esto es debido a que buena parte de las reducciones y deducciones del IRPF actual son regresivas. Son regresivas por ejemplo: las reducciones por planes de previsión, por pensiones compensatorias, o por pérdidas patrimoniales, entre otras.

\section{Cuadro 1: Valores medios en euros de algunos conceptos del IRPF, según clasificación socio-económica}

$\begin{array}{llllll}\text { Personas } & \text { Personas } & \text { Personas } & \text { Personas } & \text { Otras } & \text { Total } \\ \text { pensionistas } & \text { asalariadas } & \text { empresarias } & \text { agricultoras } & \text { personas } & \end{array}$

$\begin{array}{lcccccc}\text { Renta bruta } & 16.767,02 & 24.460,14 & 20.334,89 & 13.075,66 & 28.486,25 & 23.033,35 \\ \begin{array}{l}\text { Base imponible } \\ \text { general }\end{array} & 14.986,76 & 23.714,93 & 18.484,88 & 11.060,23 & 24.014,87 & 21.728,22 \\ \begin{array}{l}\text { Base imponible } \\ \text { ahorro }\end{array} & 1.780,26 & 745,21 & 1.850,01 & 2.015,43 & 4.471,38 & 1.305,12 \\ \text { Cuota IRPF } & 1.356,15 & 3.330,70 & 3.348,68 & 1.341,04 & 5.035,71 & 3.092,16 \\ \text { Renta ex-ante } & 15.410,87 & 21.129,44 & 16.986,21 & 11.734,63 & 23.450,55 & 19.941,18\end{array}$

Fuente: Raventós, Arcarons y Torrens (2017)

El cuadro anterior permite una primera aproximación a algunas de las características que conforman el actual IRPF. La renta bruta media de las personas asalariadas es cercana a los 24.500 euros anuales, y en las personas empresarias a 20.350 euros anuales, por tanto, las últimas declaran en promedio un 16,9\% menos de rendimientos. Si se atiende a los rendimientos integrados en la base imponible general el porcentaje anterior aumenta hasta el $22,1 \%$; sin embargo, las personas consideradas empresarias, tienen en promedio rendimientos medios de la base imponible del ahorro prácticamente 2,5 veces superiores a los de las personas asalariadas. Por último, la cuota del impuesto media es prácticamente coincidente en ambos colectivos, alrededor de los 3.350 euros anuales.

El estudio pretende financiar una RB, que por definición de universal la recibiría toda la población incluidas las personas residentes acreditadas, de una cantidad igual al umbral de la pobreza (cantidad que luego se especificará), que no estaría gravada por el IRPF (lo que significa que hasta la cantidad de RB no se paga nada por IRPF, no así a partir del primer euro que se gana por encima de la RB).

La financiación de la RB no comporta la detracción de ningún ingreso actual del Estado por la vía del IRPF. Es decir, en este estudio se recoge la misma cantidad por IRPF que la del año de referencia y además la necesaria para financiar la RB. 
La RB substituye toda otra prestación pública monetaria inferior: subsidios, becas, pensiones... En el caso de una persona que cobra una prestación monetaria pública inferior a la cantidad que representa la RB, dicha prestación se elimina y pasa a ser cubierta por la RB. En el caso de una persona que cobra una prestación superior a la cantidad que representa la $\mathrm{RB}$, su prestación se elimina hasta la cantidad de RB, pero el resto de su prestación sigue percibiéndola en los mismos términos actuales. La persona que recibe, pues, una prestación inferior a la RB, sale ganando respecto a la situación de partida. Quien recibe una prestación superior a la RB, se queda en la misma situación, no gana ni pierde respecto a la situación de partida. ${ }^{11}$

El criterio de la cantidad de RB es, según el mencionado estudio, al menos igual al umbral de la pobreza. El umbral de la pobreza que utiliza la UE: aquella persona que está por debajo del $60 \%$ de la mediana de los ingresos de la población. En el estudio se fijó en 7.471 euros anuales (622,5 en doce mensualidades) para las personas mayores de 18 años. Recuérdese que esa sería la estimación media del umbral de la pobreza para el conjunto del reino de España dejando al margen, como ya se ha dicho, la CAV y Navarra. La RB fijada para los menores de edad es de 1.494 euros anuales, una quinta parte de la cantidad para los adultos $^{12}$. Para que no quede la menor duda: al ser la RB individual, en un hogar con una persona adulta y tres menores, los ingresos por RB serían de 11.952 euros anuales, y para un hogar con tres adultos y un menor, serían de 23.907.

En el estudio se separan dos poblaciones a efectos únicamente analíticos: la que está comprendida dentro del IRPF y la que está fuera.

Primero veamos el ahorro que, según los criterios especificados, produciría esta financiación de la RB. Recuérdese que una de las características que se han apuntado de este modelo de financiación de la RB es que sustituye a cualquier otra prestación pública monetaria (si la prestación es de cuantía inferior se elimina y si la prestación es de cuantía superior, se elimina hasta la cantidad que representa la RB).

11 Si bien, el modelo econométrico permite variaciones para favorecer aún más a determinados grupos como el de algunos jubilados. Por ejemplo, que no perdiera la persona jubilada que recibiera una cantidad equivalente de tres veces la RB.

12 Lo de una quinta parte de la cantidad fijada para los adultos merece un breve comentario. El coste que supone la vida de una persona es menor que el coste de cinco personas, aunque sea en el mismo hogar. Pero no cinco veces menos. Un criterio usado por la OCDE llamado escala de Oxford es que el primer adulto de la familia computa al $100 \%$, el resto de adultos un $70 \%$ y los menores de 14 años un 50\% (aunque la OCDE también utiliza otras escalas como la llamada "OCDE modificada": el primer adulto computa también al 100\%, los adultos adicionales el 50\% y los menores de 14 años el 30\%). Así, el número de unidades de consumo (u.c.) en un hogar se calcula de la siguiente forma: $\mathrm{N}^{\mathrm{o}}$ de u.c. $=1+(\mathrm{a}-1)$ x $0,7+\mathrm{b} \times 0,5$ ( $a$ es el número de adultos y $b$ es el número de menores). Así que, en la propuesta de financiación, en un hogar con tres personas adultas y un menor, se percibiría 23.907 y, por la escala de Oxford, 21.664 euros; si bien puede haber una pequeña distorsión por las edades consideradas, 18 años en nuestro caso, 14 en el de la OCDE. Y el umbral de pobreza no deja de ser una convención (como queda dicho: el 60\% de la renta mediana de los habitantes de una zona geográfica), más relacionada con la desigualdad que con un indicador objetivo de pobreza o necesidad material. Si nos inclinamos por la RB individual y no familiar, no es por una cuestión técnica, sino porque consideramos, entre otras razones, que una RB favorece a las mujeres por el hecho precisamente de ser individual. 
Cuadro 2: Ahorros

\begin{tabular}{|l|l|}
\hline Tipo de prestación o subsidio & Importe \\
\hline Subsidios y ayudas a la Familia & $3.661,68$ \\
Subsidios y ayudas a la vivienda & $2.164,76$ \\
Subsidios de Exclusión Social & $1.957,84$ \\
Pensiones & $54.023,56$ \\
Prestaciones de desempleo & $21.405,84$ \\
Becas & $1.917,07$ \\
Clases pasivas del Estado & $3.815,71$ \\
Reservistas sin destino (FFAA y FCSE) & 258,95 \\
Sacerdotes & 126,5 \\
Reclusos & 533,57 \\
50\% gastos administrativos & $2.356,81$ \\
\hline Total & $\mathbf{9 2 . 2 2 2 , 2 9}$ \\
\hline
\end{tabular}

Fuente: Raventós, Arcarons y Torrens (2017) con datos de Eurostat, INE, Eustat, I. Estadístico de Navarra, Ministerio de Educación, Ministerio de Hacienda, MUFACE, Ministerio de Defensa, Ministerio del Interior, Conferencia Episcopal, Ministerio de Trabajo (TGSS y SPEE). Importe: millones de euros.

Es decir, habría un ahorro de 92.222,59 millones de euros.

El coste de la RB para la población no detectada por el IRPF queda pormenorizado en el Cuadro 3. La población detectada por el IRPF en el Cuadro 4.

Cuadro 3: Coste y financiación para la población no detectada en el IRPF

\begin{tabular}{|c|c|c|c|c|}
\hline & $\begin{array}{l}\text { INE } \\
\text { (personas) }\end{array}$ & $\begin{array}{l}\text { P-IRPF } \\
\text { (personas) }\end{array}$ & $\begin{array}{l}\text { P-No IRPF } \\
\text { (personas) }\end{array}$ & Importe \\
\hline Población menor 18 años & 7.819.887 & 6.515 .781 & 1.304 .106 & $1.948,59$ \\
\hline Población adulta & 35.926 .543 & 27.774.210 & 8.152.333 & $60.906,08$ \\
\hline Total & 43.746.430 & 34.289.991 & 9.456 .439 & $62.854,67$ \\
\hline \multicolumn{4}{|c|}{ Ahorro por prestaciones suprimidas } & $92.222,29$ \\
\hline \multicolumn{4}{|c|}{ Coste total RB para P-No IRPF } & $-62.854,67$ \\
\hline \multicolumn{4}{|l|}{ Remanente } & $29.367,62$ \\
\hline
\end{tabular}

Fuente: INE, AEAT y Arcarons, Raventós y Torrens (2017). Importe: millones de euros.

Obsérvese que los 92.222,59 millones de euros que sumaban todas las partidas que suponía el total de ahorro $^{13}$ de la RB, es superior en 29.367,62 millones de euros al coste

13 Con "ahorro" nos referimos a las cantidades que en la situación actual el Estado debe pagar y que no debería hacerlo con este modelo de RB. 
(que es de 62.854,67 millones de euros) de la RB para la población no detectada en el IRPF. Este remanente deberá ser recuperado, como se especificará, a la financiación de la RB correspondiente a la población comprendida en el IRPF.

Cuadro 4: Coste y objetivo de financiación para la población detectada en el IRPF

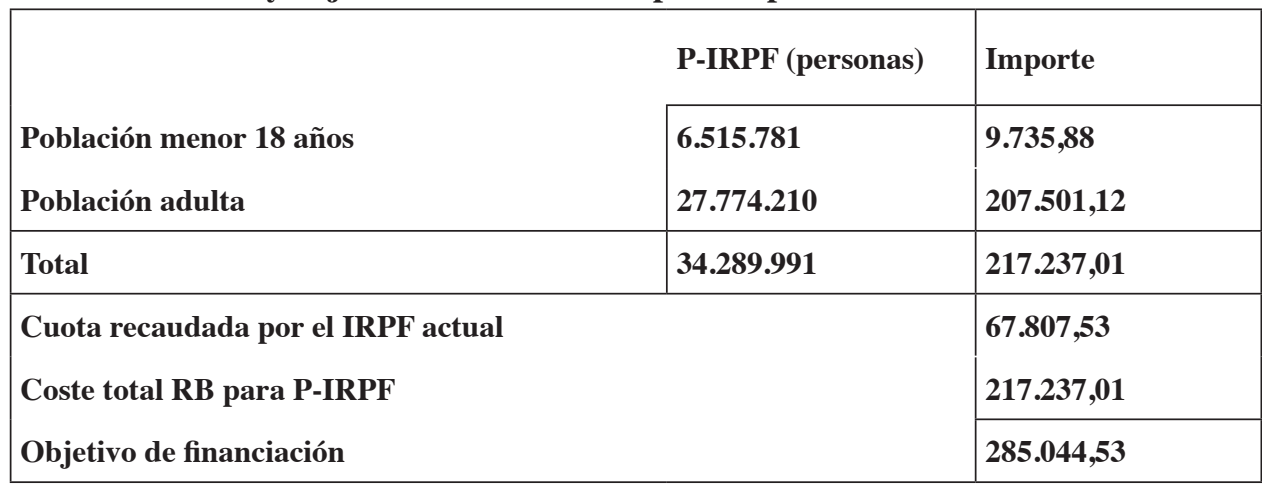

Fuente: INE, AEAT y Raventós, Arcarons y Torrens (2017). Importe: millones de euros

\section{Cuadro 5: Financiación externa}

\begin{tabular}{|l|l|}
\hline Concepto & Importe \\
\hline Remanente de P-IRPF & $\mathbf{2 9 . 3 6 7 , 6 2}$ \\
Financiación externa por Rendimientos del trabajo retenidos & $\mathbf{2 1 . 6 5 6 , 0 9}$ \\
\cline { 2 - 2 } Total & $\mathbf{5 1 . 0 2 3 , 7 1}$ \\
\hline
\end{tabular}

Fuente: AEAT y Arcarons, Raventós y Torrens (2017). Importe: millones de euros.

Las características de la reforma que se propone en el estudio son las siguientes:

1) integración de la base del ahorro en la base general;

2) eliminación de compensaciones entre rendimientos;

3) eliminación de los mínimos personales y familiares;

4) eliminación de todas las reducciones de la base (planes de pensiones, circunstancias personales, rendimientos del trabajo, etc.);

5) eliminación de todas las deducciones de la cuota (vivienda, incentivos, etc.), y

6) tipo único combinado con la transferencia de RB. ${ }^{14}$

14 El tipo único es lo que ha despertado las alarmas de más de un crítico digamos poco reflexivo al modelo explicado. Efectivamente, un tipo único sin RB puede ser muy regresivo. Como se explica a continuación con detalle, un tipo único combinado con una RB exenta de IRPF, es altamente progresivo. Adicionalmente, el modelo de simulación explicado permite la introducción de distintos tramos y tipos para la financiación de la RB. Si se concreta en el presente texto el modelo de tipo único es a efectos de simplificación. 
Este modelo de microsimulación, que es aplicable únicamente a la población detectada por el IRPF, siendo éste el motivo de la separación a efectos analíticos de las dos poblaciones según estén o no incluidas por el IRPF, permite después de la introducción de los datos anteriores extraer los siguientes resultados.

Un tipo único del $49 \%$ permite financiar la RB a más de 34 millones de personas, casi 28 millones de adultas y poco más de 6,5 millones de menores de edad, garantizando la recaudación del IRPF ex-ante que es el anterior a nuestra propuesta de reforma. También abarca a la población no detectada en el IRPF, lo que significa que los 43,7 millones de personas que conforman la población de derecho en el Reino de España percibirían la RB.

Un $61,7 \%$ de personas ganan, respecto a la situación ex-ante: pagan más impuesto por la cantidad que ganan por encima de la RB, pero lo que perciben de RB es superior al incremento en su IRPF. El porcentaje de ganadores aumenta hasta el 75\%, al añadir las personas a cargo de cada declarante, puesto que la RB es una transferencia individual. A la inversa: hay un $38,3 \%$ y un $25 \%$ de personas perdedoras, respectivamente.

El resultado se consigue con una financiación externa de 51.102 millones de euros, que se desglosan en la cantidad que hemos fijado como remanente de la financiación de la población no detectada en el IRPF (29.367,62 millones de euros) y en la aplicación del tipo único referido a las personas no obligadas a declarar (21.734,38 millones de euros).

El primer $70 \%$ de personas ordenadas por renta incrementan su participación en la renta ex-post que es la situación resultante de la implantación de la $\mathrm{RB}$, en un 9,3\% respecto a la renta ex-ante, que es la cantidad que pierde el $30 \%$ de las personas más ricas.

Se efectúa una transferencia cercana a los 32.000 millones de euros desde el $20 \%$ de las personas más ricas al primer $70 \%$ de la ordenación. En la situación ex-ante el primer $70 \%$ de la ordenación aporta el 17\% de la recaudación y el 10\% que corresponde a las personas más ricas el $53,7 \%$.

En la situación ex-post el primer $40 \%$ de personas son receptoras netas (la transferencia de RB supera la cuota aportada) y el $10 \%$ que corresponde a las personas más ricas aporta prácticamente el $64 \%$ de la nueva recaudación.

Una forma muy inmediata de visualizar quien gana y quien pierde según el nivel de riqueza es la reflejada en el siguiente gráfico 1 y en el cuadro 6.

Se observará que además de la división por decilas, se ha realizado una subdivisión en la decila más rica entre el 5 y el $2 \%$. Este gráfico es muy importante porque especifica quién gana y quien pierde, respecto a la situación de partida (ex-ante). Así, hasta la séptima decila, empezando por la más pobre, se gana con esta propuesta de financiación de la RB. Tomemos, por ejemplo, la segunda decila. Actualmente para un tipo real del 0,35\%, con la RB propuesta exenta de IRPF y con el tipo único del 49\%, su cuota es de $-59,43 \%$. Que sea negativa la cuota no significa otra cosa que se trata de una transferencia neta. Tomemos la séptima decila, como segundo ejemplo, en la cuota ex ante el tipo real es del 9,84\%, la cuota ex-post es del 6,23\%. Tomemos la decila novena, que es perdedora respecto a la situación de partida porque del $15,29 \%$ por ciento pasa al $24,32 \%$. 


\section{Gráfico 1}

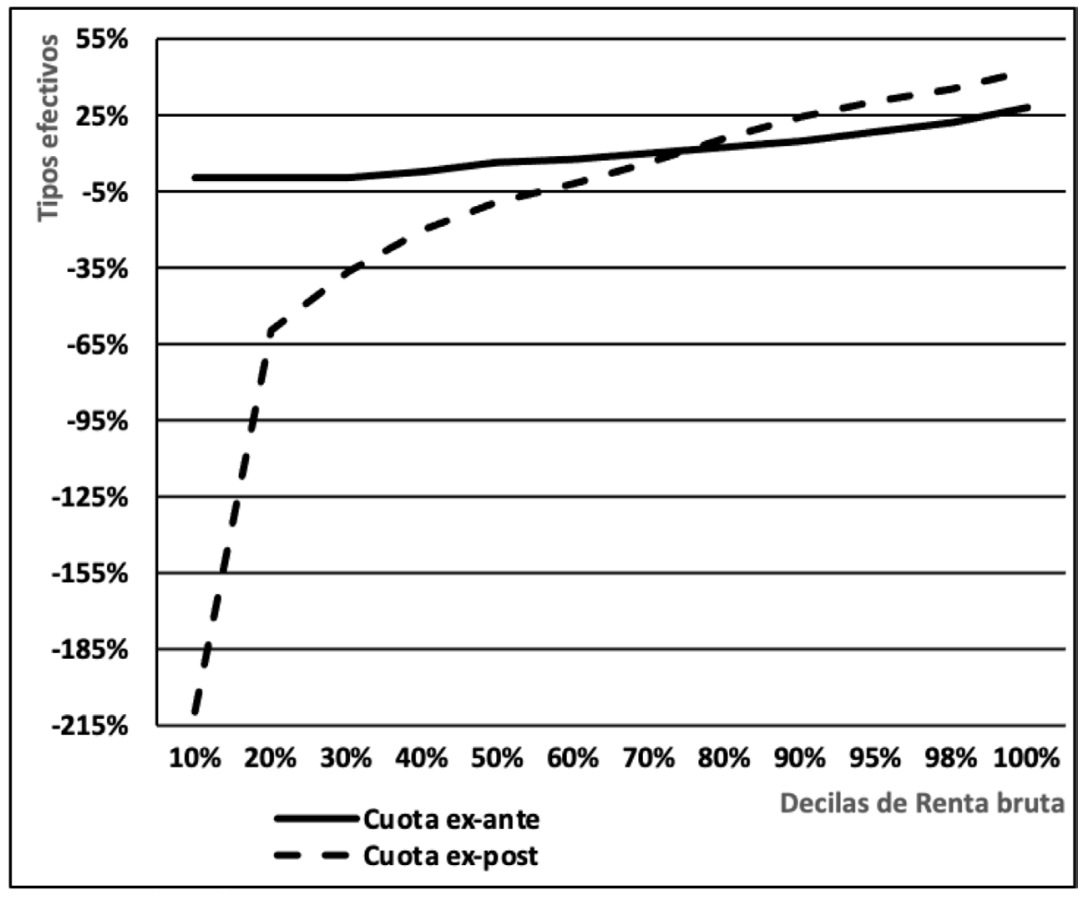

Fuente: Raventós, Arcarons, Torrens (2017). Microsimulador RB

Cuadro 6: Tipos efectivos

\begin{tabular}{|lll|}
\hline Decilas Renta bruta & Cuota ex-ante & Cuota ex-post \\
\hline $10 \%$ & $0,15 \%$ & $-209,23 \%$ \\
$20 \%$ & $0,35 \%$ & $-59,43 \%$ \\
$30 \%$ & $0,86 \%$ & $-36,77 \%$ \\
$40 \%$ & $3,28 \%$ & $-19,95 \%$ \\
$50 \%$ & $6,04 \%$ & $-9,38 \%$ \\
$60 \%$ & $7,86 \%$ & $-1,72 \%$ \\
$70 \%$ & $9,84 \%$ & $6,23 \%$ \\
$80 \%$ & $12,53 \%$ & $15,56 \%$ \\
$90 \%$ & $15,29 \%$ & $24,32 \%$ \\
$95 \%$ & $18,38 \%$ & $29,98 \%$ \\
$98 \%$ & $22,07 \%$ & $35,02 \%$ \\
$100 \%$ & $28,03 \%$ & $42,56 \%$ \\
\hline
\end{tabular}

Fuente: Arcarons, Raventós, Torrens (2017). Microsimulador RB 
Así que, como muestra el Gráfico 1 y el Cuadro 6, el 70\% de la población detectada por el IRPF sale ganando respecto de la situación actual y el $30 \%$ más rico, claro está, resulta perdedor. Y debe recordarse que la población no detectada por el IRPF también es ganadora por razones obvias: si no declara el IRPF es que está en el umbral de unas cantidades tan pequeñas que no están obligados a declarar. Puede haber alguna excepción muy extraña, pero el grueso de la población no detectada por el IRPF está bajo las condiciones señaladas. Se trata, como debe resultar evidente, de una redistribución de la riqueza de una parte que corresponde al $20 \%$ de la población más rica al $80 \%$ por ciento restante. Esto se traduce en una reducción muy significativa del índice de Gini. Si antes del IRPF actual, como se ha apuntado anteriormente, este índice es del 0,4114, y después del IRPF actual pasa a ser del 0,3664 , con la reforma propuesta pasa a ser del 0,2502. Es decir, queda reducido a más de 11 puntos..$^{15}$

Podemos resumir brevemente los muchos resultados y datos de esta modelo de financiación:

1) Se puede financiar una RB igual al umbral de la pobreza sin detraer ni un céntimo de la recaudación impositiva previa a la reforma que proponemos. Sanidad y educación públicas no quedarían afectadas ni por un céntimo, ni tan sólo otras partidas que cualquier proyecto racional debería suprimir. En el más extremo ejercicio de realismo económico, este modelo intenta demostrar que con datos oficiales y a partir únicamente del IRPF se puede financiar una RB.

2) Esto significa que en el momento de la implantación de la RB que se propone, la pobreza queda erradicada. ${ }^{16}$ Toda persona residente acreditada o ciudadana recibe una RB igual al umbral de la pobreza.

3) Que toda persona ciudadana o residente acreditada reciba una RB no es equivalente a afirmar que todas las personas resultan ganadoras en términos netos. Como creemos haber mostrado con detalle, el $20 \%$ más rico, aunque por definición recibe la RB, es la parte de la población que perdería respecto a la situación de partida. El restante $80 \%$ empezando por la persona más pobre, resultaría ganadora con la RB y la forma de financiarla aquí expuesta.

4) La redistribución de la renta resultante, como distintos indicadores muestran entre ellos el más conocido de Gini, sería mucho menos desigualitaria que la actual.

Esta financiación de la RB está basada en lo fundamental en una reforma del IRPF. Pero también puede ser complementada mediante una carga impositiva a la riqueza, un concepto más amplio que el de renta. Pero antes de aportar algunos datos económicos, es el momento de introducir la fundamentación de la Renta Máxima.

15 Otros indicadores de progresividad, como los índices Kakwani y Suits, confirman la gran progresividad de la reforma propuesta.

16 Hay quien ha objetado que, al variar el umbral de la pobreza con esta propuesta, habría igualmente un porcentaje de personas por debajo de dicho umbral y, en consecuencia, no acabaría con ella. Es una objeción interesante porque denota una forma de entender la financiación de la RB que no es la propuesta, pero resulta errónea porque matemáticamente es perfectamente posible mostrar que todo el mundo esté por encima del $60 \%$ de la mediana. Se trata de una gran redistribución de la renta y, por tanto, el umbral no tiene porqué moverse. Si se supone erróneamente que se trataría de seguir igual como hasta ahora y además añadir la RB, sí que sería correcta esta interpretación. Pero no es el caso. 


\section{Renta Máxima: Un maximum compatible con una vida republicana normal}

Hay muchos argumentos que pueden esgrimirse a favor de una Renta Máxima (RM), entre ellos que la riqueza excesiva es una fuente infinita de corrupción y venalidad porque sólo los muy ricos tienen suficiente capacidad para comprar gobiernos; que la concentración de la riqueza termina destruyendo a la democracia; que la gran concentración de la riqueza produce codicia y deterioro medioambiental, entre otros muchos que bien narra Sam Pizzigati (2018).

Como hemos dicho antes, pensamos que hay un argumento republicano contundente para justificar una RM, a saber: puesto que la riqueza y la propiedad privada son un producto esencialmente político y social, una república democrática debe ser capaz de diseñar algunos instrumentos -como una RM- que eviten que ésta quede concentrada en unas pocas y confiscatorias manos, y debe impedir también que estas manos puedan disputarle a la república su capacidad para definir el bien común y para garantizar una vida republicana y democrática normal, para todos (Casassas y Raventós, 2018). La propiedad ha estado muy concentrada a lo largo de los siglos XIX, XX y lo que llevamos del XXI, si bien con intensidades diferentes. A partir de las décadas de los 80 y 90 del siglo pasado en muchos lugares del mundo la propiedad se ha concentrado de forma nunca vista hasta el momento (Piketty, 2019).

$\mathrm{Ni}$ duda cabe que algunos regímenes políticos liberales han sido exitosos en muchos sentidos, entre otros en imponer ciertas creencias ubicuas y profundamente arraigadas, por ejemplo, que un sistema democrático de gobierno debe abstenerse de interferir en los derechos de propiedad existentes, incluso cuando estos derechos sean incompatibles con la garantía de un derecho a la existencia de los no propietarios e incluso pobres, que suelen ser mayoría. ${ }^{17} \mathrm{El}$ principal argumento para respaldar tal mantra consiste en tejer una ceñida ligazón entre la propiedad y la libertad, entendida ésta como una libertad "personal" o autonomía, que permitiría a los propietarios llevar a cabo libremente sus planes de vida sin interferencias por parte de otros, independientemente de que esta interferencia provenga de los poderes privados o públicos y, también, de si esos poderes públicos se ejercen de manera legítima o ilegítima. En paralelo con este argumento, se suele apelar a otro concepto que goza de un prestigio normativo tan indudable como el de libertad: la soberanía del propietario. El individuo propietario parecería poder gozar de una inmunidad que le confiere una soberanía personal en tanto propietario, otorgándole una autoridad exclusiva sobre la cosa y sobre los accidentes que se derivan de la propiedad de la cosa.

Lo cierto es que, pensadas las cosas de esta manera, una república sería equivalente a una asociación privada de individuos que se limitan a perseguir sus ventajas particulares mutuas, una colección de seres humanos juntados de cualquier manera y en vistas al bien privado, con todas las consecuencias que de ahí se siguen. Y una de las consecuencias de pensar de ese modo a la comunidad política sería eliminar la crucial diferencia conceptual entre la negociación colectiva y la deliberación pública. En una negociación las preferencias de las partes están congeladas a lo largo del proceso y cada cual busca promover su mejor ventaja aportando razones privadas. Pero sería por cierto absurdo, por ejemplo, que un diputado en

17 Utilizamos el término "derecho a la existencia" en el sentido revolucionario francés y específicamente en Robespierre: "la sociedad debe garantizar a todos sus miembros como primer derecho, el de existir material y socialmente". Para este tema, Raventós (2007) y Laín (2020). 
el parlamento defendiera un proyecto de ley argumentado que esa ley beneficiaría mucho a su familia (Bertomeu, 2016).

Esta concepción de la riqueza y la propiedad entendida como un ámbito de autonomía individual absoluta separada del colectivo, y que pretende incluso competir con el poder soberano delegado en los gobiernos democráticos, es claramente incompatible con una concepción republicana fiduciaria y democrática de la propiedad. No hay que olvidar que asignar derechos de propiedad absolutos a algunos y no a otros o a todos -y muy especialmente sobre objetos físicos externos que son rivales y excluyentes- necesariamente implica incumplir con el deber de velar por los derechos y necesidades de todos. Según la concepción republicana y fiduciaria del poder político, los gobiernos y sus mandatarios o comisarios sólo son un trustee, un fideicomisario del pueblo libre que es el fideicomitente. Se trata de una relación asimétrica en la que el pueblo fideicomitente siempre puede romper de manera unilateral su relación con la autoridad fideicomisaria, con sólo manifestar que ha perdido la confianza en ella. Ahora bien, para una concepción republicano-fiduciaria de la soberanía política, sin duda alguna, la meta es promover la libertad como no dominación por parte de los poderes públicos y privados. Una de las principales notas propias de la concepción republicana fiduciaria del poder político es que quien ejerce un dominium sobre objetos rivales amparado en una supuesta soberanía absoluta sobre su propiedad, también tiene la capacidad de ejercer imperium poniendo a sus órdenes a los poderes públicos y a sus conciudadanos, de ahí que el republicanismo también abone una concepción fiduciaria de la propiedad en la que el principal es el soberano. ${ }^{18}$

Por tanto, según la concepción fiduciaria y republicana del poder político, no se trata sólo de evitar interferencias arbitrarias, de proteger al vulnerable o de prevenir males ex post; porque la república tiene el deber de promover activamente la no dominación por parte de los poderes públicos y privados ex ante, en la medida en que tales poderes atenten $-\mathrm{O}$ tengan la capacidad de hacerlo- con los derechos constitutivos de todos, comenzando por el derecho básico a la libertad, la capacidad de ser un individuo que goce de una existencia social autónoma. Y es en este sentido que una teoría republicana y democrática ofrece herramientas normativas propias para pensar en una soberanía política fiduciaria con legitimidad para regular los institutos jurídicos de la propiedad privada y pública, que impidan la universalización de la libertad no dominada.

En el modelo de financiación de una RB anteriormente presentado, se mencionaba que aunque estaba basado fundamentalmente en una reforma del IRPF, también podía ser complementado mediante una carga impositiva a la riqueza. Si se implantase un impuesto del $10 \%$ a la decila más rica en patrimonio de la población del Reino de España (sin contar su vivienda de residencia), podrían obtenerse más de 96.000 millones de euros. Exactamente sería aplicar al cruce de la decila de mayor patrimonio (riqueza) y de la decila de mayor renta, que según la Encuesta Financiera de las Familias del año 2014 acumula las nada despreciables cifras de

18 La concepción republicana y fiduciaria de la soberanía política se asienta en una larga tradición jurídicopolítica, entre ellas la ciceroniana. Huelga decir que en el mundo moderno, el Locke maduro del Second Treatise abrazó la causa radical de los Levellers ingleses proponiendo una defensa de la soberanía del pueblo en términos de una relación fiduciaria entre principal-agente. Véase para este tema Mundó (2017). Entre los autores contemporáneos que rescatan una concepción republicana fiduciaria de la soberanía política, desde una perspectiva jurídico y filosófico-política: Pettit (1997) y Fox-Decent (2012), entre otros. 
más de 0,15 billones de euros de renta y de 0,96 billones de euros de patrimonio (descontando la vivienda habitual).${ }^{19}$ Mucho más dinero que todas las pensiones contributivas y no contributivas juntas. Y solamente se trataría del 10\%. Un $20 \%$ al $10 \%$ más rico patrimonialmente recaudaría claro está el doble, es decir, 192.000 millones. Aun así, este 10\% de la población seguiría siendo rica en patrimonio, sin lugar a dudas. Otro ejemplo: también podría implantarse un impuesto según el nivel de riqueza, tal como propone Piketty en su último libro Capital e ideología: a quien disponga de 100 veces el patrimonio medio, se le aplicaría un tipo del 10\%; a quien lo tenga de 1.000 veces, del $60 \%$; a quien lo tenga de 10.000 veces, del $90 \%$. No hace falta precisar que tales imposiciones no serían exactamente una renta máxima, que en realidad sería una tasa marginal impositiva del $100 \%$ a partir de determinada cantidad de riqueza, pero esta última imposición del 90\% se acercaría (Raventós, 2020).

Debe recordarse que la propiedad está muy concentrada, mucho más que la renta. Así, por ejemplo, la decila superior tanto en Europa como en los Estados Unidos acumula en la actualidad más del $55 \%$ y del $70 \%$, respectivamente de los activos inmobiliarios y financieros. Y el centil superior más del $20 \%$ y del $40 \%$, respectivamente (Piketty, 2019, capítulo 10). Solamente el $1 \%$ de la población acumula estos porcentajes de riqueza. Y la opacidad informativa sobre la riqueza en todo el mundo es muy grande, algo que afecta a la democracia de una forma no señalada muy a menudo. Se trata de una desproporción y concentración solamente superadas, desde que se tienen datos comparables, en las primeras décadas del siglo XX.

Esta concentración de la propiedad no se traduce solamente en una gran desigualdad, por importante que esta sea, sino en una capacidad de los grandes poderes privados para imponer a los Estados su concepción del bien público. Imposición que se traduce en una pérdida de la libertad para la inmensa mayoría no ultrarrica. Como hemos dicho antes, esta situación es inaceptable desde un punto de vista republicano y democrático. "También ahora (...), las dinastías empresariales y los grandes imperios privados económicos vuelven a desafiar con éxito el derecho inalienable de las Repúblicas a determinar el bien público" (Domènech, 2006, 17). La Renta Básica y la Renta Máxima pueden ser, sin duda alguna, dos herramientas legítimas para conseguir una libertad republicana para toda la población. No como únicas medidas, pero sí como componentes principales de una política económica y social republicanas.

\section{Bibliografía}

Arcarons, J., Domènech, A., Raventós, D. y Torrens, L. (2014), “Un modelo de financiación de la Renta Básica para el conjunto del Reino de España: sí, se puede y es racional", https://www.sinpermiso.info/textos/un-modelo-de-financiacin-de-la-renta-bsica-para-elconjunto-del-reino-de-espaa-s-se-puede-y-es, recuperado 7 de mayo de 2020.

Arcarons, J., Raventós, D. y Torrens, L. (2017), Renta Básica Incondicional. Una propuesta de financiación racional y justa, Barcelona: Serbal.

Bertomeu, M.J. (2016), “La declaración de derechos humanos de 1948 ¿un retorno de la concepción republicana (y fiduciaria) de los derechos constitutivos?”, en Britos, P., Erazun, F. (eds.), Miradas sobre la Justicia Global, Neuquén, República Argentina: Editorial Universitaria de la Universidad Nacional del Comahue.

19 Agradecemos a Jordi Arcarons la cesión de estos datos. 
Bertomeu, M.J. (2016), “Bien común”, en: Pereda, C. (ed.), Diccionario de Justicia, México: Siglo Veintiuno.

Casassas, D. (2018), Libertad incondicional. La renta básica en la revolución democrática, Madrid: Paidós.

Casassas, D., Raventós,D. (2018) “La viabilidad de la Renta Básica en el Reino de España”, https://www.sinpermiso.info/textos/la-viabilidad-de-la-renta-basica-en-el-reino-deespana. Recuperado 20 de junio de 2020

Cicerón (1989), Sobre los deberes, trad. y notas de J.G. Cabañero, Madrid: Tecnos.

Domènech, A. (2019), El eclipse de la fraternidad. Una revisión republicana de la tradición socialista, Madrid: Akal.

Domènech, A. (2006), "República y socialismo, también para el siglo XXI", Sin Permiso, 1, pp. 17-46.

Fox-Decent, E. (2012), Sovereignty's Promise: The State as Fiduciary, Oxford: Oxford University Press.

Gauthier, F. (1992), Triomphe et mort du droit naturel en Révolution 1789-1795-1802, Paris : PUF.

Gourevitch, A. (2015), From Slavery to the Cooperative Commonwealth, Labor and Republican Liberty in the Ninteenth Century, Cambridge: Cambridge University Press.

Hammel, C. (2019), "Le républicanisme des droits. Enjeux conceptuels d'un passé utile", https://hal.archives-ouvertes.fr/hal-02327704, recuperado 20 de abril de 2020.

Laín, B. (2020), "Del derecho natural al pacto fiduciario: gobierno y propiedad en la economía política republicana”, Isegoría, 62, pp. 9-34.

Mundó, J. (2017), "La constitución fiduciaria de la libertad política. (Por qué son importantes las coyunturas interpretativas en la filosofía política)", Isegoría, 57, pp. 433-454.

Murray, Ch. (2006), In Our Hands: A Plan To Replace The Welfare State, Washington: AEL Press.

Nadal, A. (2019), "El fracaso histórico del capital”, https://www.sinpermiso.info/textos/elfracaso-historico-del-capital, recuperado 28 de abril de 2020

Pettit, P. (1997), Republicanism. A Theory of Freedom and Government, Oxford University Press: Nueva York.

Piketty, T. (2019), Capital i ideologia, Barcelona: Edicions 62.

Pizzigati, A, (2018), The Case for a maximum Wage, Cambridge: Polity Press.

Raventós, D. (2020), "Darwin, robespieristas, realistas, radicales y la 'paguita'”, https:// www.sinpermiso.info/textos/darwin-robespieristas-realistas-radicales-y-la-paguita, recuperado el 7 de mayo de 2020.

Roberts, C.W. (2017), Marx's Inferno. The Political Theory of Capital, Princeton: Princeton University Press.

Szlinder, M. (2018), "Entrevista sobre renta básica y emancipación social”, Sin Permiso, 16, pp. 117-124. 
